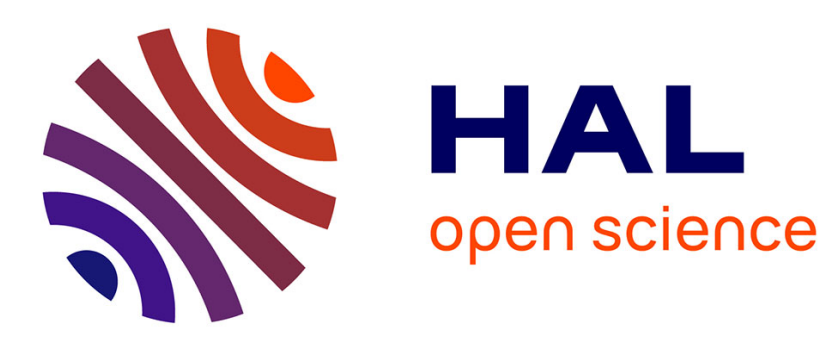

\title{
Effets de la sylviculture et de l'exploitation forestière sur la diversité végétale.
}

\author{
Marc Deconchat, Gérard Balent
}

\section{To cite this version:}

Marc Deconchat, Gérard Balent. Effets de la sylviculture et de l'exploitation forestière sur la diversité végétale.. Revue forestière française, 2002, 54 (6), pp.559-566. 10.4267/2042/4943 . hal-03449305

\section{HAL Id: hal-03449305 https://hal.science/hal-03449305}

Submitted on 25 Nov 2021

HAL is a multi-disciplinary open access archive for the deposit and dissemination of scientific research documents, whether they are published or not. The documents may come from teaching and research institutions in France or abroad, or from public or private research centers.
L'archive ouverte pluridisciplinaire HAL, est destinée au dépôt et à la diffusion de documents scientifiques de niveau recherche, publiés ou non, émanant des établissements d'enseignement et de recherche français ou étrangers, des laboratoires publics ou privés. 


\title{
EFFETS DE LA SYLVICULTURE ET DE L'EXPLOITATION FORESTIÈRE SUR LA DIVERSITÉ VÉGÉTALE
}

\author{
Marc Deconchat - Gérard Balent
}

Depuis longtemps, les forestiers cherchent à gérer la diversité de la végétation, celle des arbres bien sûr, mais aussi celle des autres espèces végétales. Les raisons sont sylvicoles, patrimoniales et fonctionnelles. La limitation de la concurrence avec les arbres, surtout dans les stades jeunes, reste une des motivations premières de la gestion de la végétation forestière (voir Frochot et al. dans ce numéro). S'ajoutent aujourd'hui des raisons patrimoniales de conservation d'espèces végétales rares ou menacées poussant en forêt (Rameau et Timbal, 1987). Un exemple bien connu est celui du Sabot de Vénus (Cypripedium calceolus) dont la protection implique par endroits des gestions forestières et des modes d'exploitation particuliers. D'autres raisons sont maintenant avancées, touchant au fonctionnement des écosystèmes forestiers. Ainsi, le rôle de la végétation herbacée pour réduire les pertes par lessivage après les coupes rases est mis en avant (Mou et al., 1993). Le rôle de la végétation pour des espèces animales est aussi une raison incitant à une meilleure gestion de la flore, soit pour favoriser ces espèces, soit pour réduire leur développement (Bergquist et al., 1999). On peut ainsi retenir l'intéressante expérience conduite actuellement par l'INRA dans le massif landais où l'on teste l'introduction de taches de feuillus dans les Pins maritimes afin de voir si elle réduit la pression des ravageurs et augmente la diversité des espèces auxiliaires.

La gestion de la flore nécessite de connaître les facteurs qui l'influencent et notamment ceux sur lesquels il est possible d'agir (Le Tacon et al., 2001). Parmi les facteurs très difficilement modifiables par la gestion, on peut citer la situation biogéographique, les conditions stationnelles (Chevalier et al., 2001), l'histoire, l'influence des milieux environnants (source de colonisation pour certaines espèces : voir Dupouey et al. dans ce numéro). Parmi les facteurs sur lesquels l'homme peut agir, on peut distinguer :

- des facteurs sylvicoles influant principalement sur la disponibilité en lumière et en eau, et sur le régime de perturbation déterminé par la fréquence des coupes (Halpern et Spies, 1995);

- des facteurs liés à l'exploitation forestière et aux perturbations particulières qu'elle occasionne au niveau du sol (Deconchat et Balent, 2001a);

- l'influence des grands herbivores (Bergquist et al., 1999);

- et, enfin, les autres usages de la forêt comme sa fréquentation (Kobayashi et al., 1997).

Une connaissance de l'importance respective de ces groupes de facteurs est nécessaire pour gérer la flore. Ils concernent en effet des gestionnaires et des opérateurs différents : le sylviculteur, l'exploitant forestier, le chasseur et les autres usagers. La sylviculture est le facteur qui a reçu jusque-là le plus d'attention, mais les autres facteurs peuvent être importants aussi. 
L'objectif est ici de présenter et de mettre en relation les résultats de deux études concernant, d'une part, les effets de la sylviculture sur la flore dans les taillis avec réserves des forêts fragmentées du Sud-Ouest de la France et, d'autre part, les effets des pratiques d'exploitation forestière (Deconchat, 1999).

\section{LA VÉGÉTATION DES TAILLIS AVEC RÉSERVES DU SUD-OUEST DE LA FRANCE}

Les forêts des coteaux de Gascogne sont fortement fragmentées et sont souvent gérées par des agriculteurs, essentiellement sous forme de taillis à rotation de 20 à 40 ans, avec une densité faible de réserves (o à 150 arbres/ha) conservées pour produire du bois d'œuvre (Balent, 1996). Une partie du taillis est destinée à l'usine de pâte à papier de Saint-Gaudens qui a demandé à I'INRA-URSAD de Toulouse d'étudier les facteurs affectant la biodiversité, notamment au niveau de la végétation, de manière à mieux maîtriser, dans la perspective d'une certification, les conséquences de leurs pratiques (Deconchat et al., 1998 ; Deconchat, 1999).

Pour étudier l'effet de la gestion en taillis avec réserves sur la flore, nous avons établi un dispositif d'observation constitué de 96 placettes de $400 \mathrm{~m}^{2}$ dans des parcelles différant par l'âge du taillis (depuis de jeunes rejets jusqu'à des peuplements en âge d'être exploités), la densité de réserves (allant de o, c'est-à-dire d'un taillis pur, à plus de 200 réserves par hectare) et la proximité des parcelles agricoles. D'autres variables descriptives des conditions du milieu ont été relevées. Ce dispositif a été utilisé pour étudier conjointement la végétation, les oiseaux et les collemboles (insectes du sol). Les analyses ont été limitées aux espèces non ligneuses ayant un renouvellement annuel de leurs parties aériennes, considérées comme potentiellement les plus sensibles aux effets de la sylviculture.

Le groupe d'espèces retenu comporte 187 espèces (62 espèces non retenues), dont 151 avec une fréquence d'observation inférieure à $10 \%$. Le Tamier commun (Tamus communis) est la seule espèce dont la fréquence dépasse $50 \%$. On peut noter l'observation de Lys martagon (Lilium martagon) comme espèce à valeur patrimoniale. La richesse spécifique moyenne par placette passe de 8 espèces du groupe étudié dans les stades matures à 16 espèces dans les parcelles récemment coupées avec une forte variabilité. La richesse s'accroît sans perte puisque les espèces des stades matures sont toujours présentes dans les coupes. La communauté végétale des coupes est plus instable, avec plus d'espèces annuelles et anémochores (dont les graines sont disséminées par le vent), alors que les plantes géophytes, hivernant dans le sol, sont plus nombreuses dans les stades matures. Beaucoup d'espèces n'ont été observées que dans les coupes, mais leur fréquence est souvent trop faible pour que leur soient appliqués des tests statistiques. Il s'agit d'espèces rudérales (provenant des milieux agricoles) et aptes à se développer rapidement dans des conditions perturbées (exemple : Cirsium arvense, Solidago canadensis, Taraxacum officinale).

La richesse spécifique végétale décroît rapidement après la coupe ; elle est minimale entre 3 et 10 ans avec 5 espèces en moyenne par placette. Les espèces présentes uniquement dans les coupes disparaissent progressivement au cours de cette période. La richesse spécifique semble augmenter ensuite pour atteindre environ 8 espèces par placette en moyenne. Hypericum pulchrum, Hypericum perforatum, Solidago canadensis, Centaurium erythrea, Cirsium arvense voient leur fréquence d'apparition se réduire très significativement au cours du cycle sylvicole. Les résultats montrent donc une opposition très nette entre la végétation des coupes et celles des autres stades entre lesquels les différences sont plus subtiles. 
La densité de réserves conservées lors de la coupe n'a pas d'effet sur la richesse botanique, quelles que soient les classes d'âge. Tout au plus peut-on identifier un effet des fortes densités de réserves sur la composition botanique dans le sous-groupe des coupes.

Dans une analyse multivariée sous contraintes (figure 1, ci-dessous), après une sélection des variables les plus explicatives, qui n'a pas retenu la densité de réserves dont la contribution était la plus faible de toutes les variables, le premier facteur explicatif de la composition botanique est le temps écoulé depuis la dernière exploitation (TEDDE), c'est-à-dire l'âge des brins du taillis, très corrélé à la fermeture de la canopée (ST16). Le type de station apparaît comme important, mais la faible variabilité stationnelle dans l'échantillon limite son expression. Le second facteur explicatif de la composition botanique est la distance à la plus proche lisière du bois (BDL), c'est-à-dire l'influence du milieu agricole. On observe que ce facteur introduit une forte variabilité de la composition botanique, représentée sur le second axe du plan factoriel, surtout dans la partie correspondant au stade jeune des peuplements. Cela signifie que l'influence de la proximité des terres agricoles sur la flore forestière s'exprime surtout dans les coupes et s'atténue ensuite dans les stades matures qui présentent une végétation moins variable, quelle que soit leur localisation. Dans les placettes coupées et proches de la lisière, on observe des espèces fréquentes aussi dans les cultures, alors que, dans les placettes situées à l'intérieur des forêts, on observe des espèces plus forestières.

\section{FIGURE 1 PLAN FACTORIEL DE L'ANALYSE FACTORIELLE DES CORRESPONDANCES SOUS CONTRAINTE DE VARIABLES INSTRUMENTALES}

Les espèces sont celles qui ont une fréquence supérieure à $10 \%$; elles sont codées avec les 3 premières lettres du nom de genre d'une part, d'espèce d'autre part : TEDDE : temps écoulé depuis la dernière exploitation ; ST16 : densité de végétation dans la strate 8-16m PROP : type de propriétaire ; STAT : station forestière ; BDL : distance à la plus proche lisière

Les points correspondent à la position des placettes dans le plan factoriel

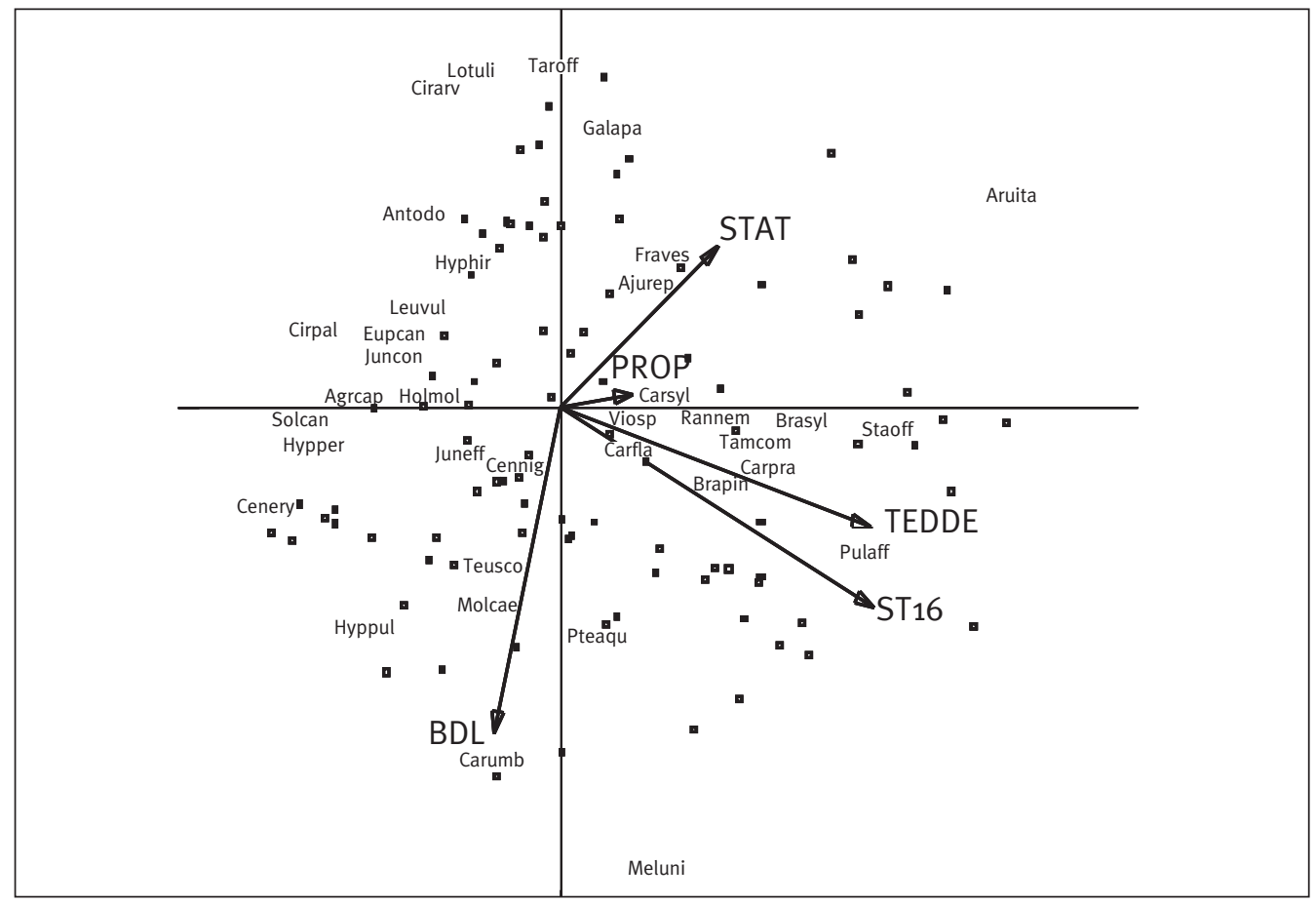


Ces résultats montrent que les perturbations occasionnées par la coupe sont une cause importante des variations de la végétation forestière. Celle-ci provoque l'arrivée de nouvelles espèces en forêt, sans qu'il y ait apparemment de disparition, contrairement à ce qui a pu s'observer dans d'autres situations (Halpern et Spies, 1995). Par contre, les variations dues aux pratiques sylvicoles, notamment la densité de taillis, ne semblent pas avoir d'effet sur la végétation, tout au moins dans les conditions de l'étude (Deconchat et Balent, 2001b). Les densités de réserves de l'échantillon sont sans doute trop faibles pour qu'on puisse mettre en évidence leur influence sur la disponibilité en lumière dans la coupe par exemple ; la fermeture rapide du couvert par le taillis constitue un phénomène beaucoup plus important que celui de la présence des arbres de réserve. L'influence d'un facteur spatial comme la distance à la lisière la plus proche démontre que les pratiques sylvicoles ne sont pas les seuls facteurs influant sur la végétation. L'influence des milieux environnants a été longtemps négligée comme facteur explicatif. Dans les forêts fragmentées de la zone d'étude, ce facteur ne peut être omis et son influence prépondérante est avérée. Ce résultat débouche sur une approche différente de la gestion de la végétation forestière selon que l'on est dans une grande forêt, loin des lisières, là où les espèces forestières dominent, ou que l'on est dans une petite forêt, en un lieu proche de la lisière, là où des risques de colonisation par des espèces agricoles peuvent poser des problèmes.

\section{EFFETS SUR LA FLORE DES PERTURBATIONS DE LA SURFACE DU SOL CAUSÉES PAR L'EXPLOITATION}

Pour étudier les effets de l'exploitation, une étude a été conduite qui compare la végétation de placeaux de petite surface $\left(1 \mathrm{~m}^{2}\right)$ dans 4 situations : un peuplement de taillis-sous-futaie mature (TSF), trois zones coupées depuis 2 ans, non perturbées par l'exploitation (sol intact), perturbées par l'exploitation (sol perturbé) ou couvertes de rémanents (bois abandonnés par l'exploitant) (Deconchat et Balent, 2001a). L'analyse de la répartition des 75 espèces observées entre les 4 modalités démontre, comme l'observation directe le suggérait, qu'il y a des différences de richesse et de composition botaniques très grandes (figure 2, p. 563), certaines espèces n'étant observées que dans une modalité, alors que d'autres l'ont été dans les 4 . Les espèces du peuplement avant exploitation (TSF) se retrouvent dans les coupes, à 5 exceptions près, constituées par des plantes de très faible fréquence. Cependant, elles ne se trouvent pas dans toutes les modalités d'état du terrain de la coupe, 4 espèces étant absentes d'au moins une modalité. La coupe favorise l'arrivée de 13 espèces, quelles que soient les modalités, mais la perturbation du sol favorise à elle seule 29 espèces, ayant cependant chacune une très faible fréquence. La richesse spécifique moyenne des placeaux (moyenne du nombre d'espèces observées par placeau) augmente fortement de 6 espèces (+ $120 \%$ ) là où le sol a été perturbé par rapport aux situations non perturbées qui sont néanmoins plus riches que les peuplements de taillis-sous-futaie avant exploitation (figure 3, p. 563).

L'état de surface de la coupe constitue donc, avec la mise en lumière, un facteur de diversification de la végétation très important. Les zones perturbées ont une végétation très diversifiée mais peu forestière, composée d'espèces anémophiles ; les zones intactes sont moins diversifiées mais plus forestières, alors que les zones à rémanents possèdent la végétation la plus proche de celle des zones de référence non exploitées.

Les trois types de végétation issus de l'exploitation suivent des trajectoires différentes qui convergent apparemment vers la flore observée dans les stades matures. La compétition pour la lumière, contrôlée par le développement de la structure arborée, devient alors le facteur prépondérant ; mais les traces des perturbations (tassement, ornières ou gros bois morts) peuvent 
maintenir leur influence longtemps. Entre 5 et 10 ans, le taillis atteint un développement suffisant pour fermer la canopée et réduire fortement la lumière au sol (Barkham, 1992 ; van der Werf, 1991). La croissance des arbres de réserve et l'étagement de la canopée du taillis après une vingtaine d'années vers les stades matures permettent une diversification lente de la végétation par l'installation de quelques espèces nouvelles.

FIGURE 2 COMPARAISON DES FLORES SELON LES 4 MODALITÉS

La richesse en espèces dans chaque modalité est découpée en segments de longueur proportionnelle à la richesse en espèces communes entre modalités ou restreinte à une modalité.

Par exemple, 12 espèces ont été observées dans les 4 modalités, alors que seulement 5 l'étaient uniquement dans le taillis-sous-futaie

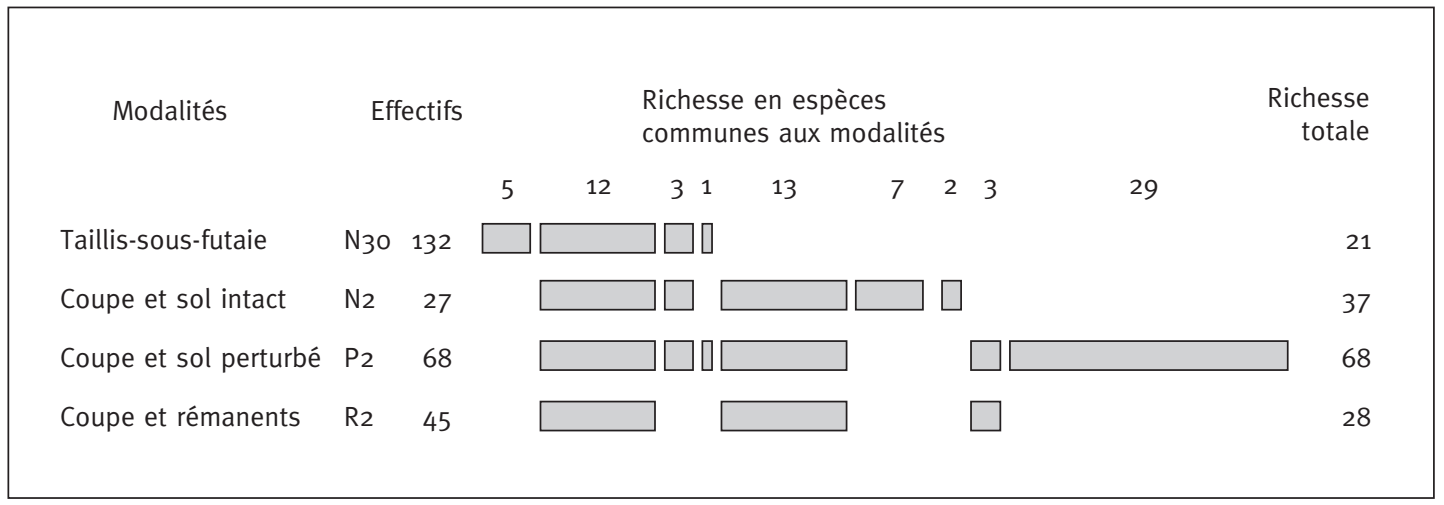

FIGURE 3

NOMBRE D’ESPĖCES DE PLANTES DANS LES CARRÉS D'UN MÈTRE CARRÉ EN FONCTION DE L'ÉTAT DE SURFACE ENGENDRÉ PAR L'EXPLOITATION FORESTIÈRE

$N$ : intact ; $P$ : perturbé ; $R$ : rémanent (Deconchat, 1999)

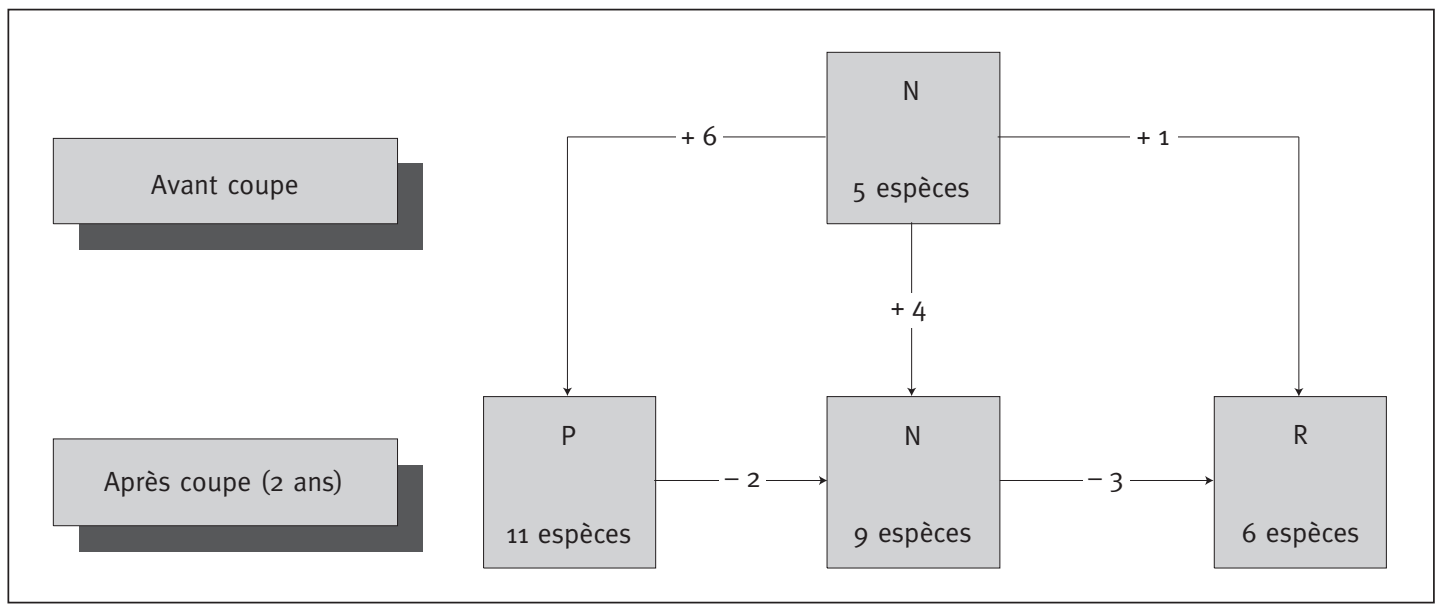




\section{DISCUSSION ET CONCLUSIONS}

Bien qu'elle ait été beaucoup étudiée, la dynamique de la végétation reste difficile à prévoir et des recherches sont toujours nécessaires. Les résultats indiquent deux directions de recherche qui ont peu été abordées jusqu'alors. La première concerne l'influence de la dimension spatiale dans la dynamique de la végétation. L'approche par les stations forestières, qui fait correspondre une station à un type de végétation, prend sans doute insuffisamment en compte la dimension spatiale. Où que soit localisée la station par rapport à d'autres formations végétales, elle est censée comporter le même cortège floristique. Or, les résultats montrent que, après une même perturbation - la coupe - des flores très similaires suivent des trajectoires différentes qui dépendent apparemment surtout de la localisation des parcelles dans la forêt. Cette influence s'exprime bien au-delà de l'effet de lisière connu et facilement visible sur le périmètre des forêts où l'on observe un ourlet d'espèces héliophiles. L'importance de ces facteurs spatiaux est probablement plus faible dans les grands massifs que dans les forêts fragmentées comme celles étudiées, car les conditions internes sont "tamponnées" dans ces grandes forêts par rapport aux espaces agricoles. Cependant, dans la perspective de changements rapides des conditions bioclimatiques, la dimension spatiale de la dynamique de la végétation prendra une importance accrue car les modifications de la flore dépendront en partie de la disponibilité et de la proximité de sources de colonisation par des espèces mieux adaptées aux nouvelles conditions.

La seconde direction de recherche qui émerge concerne les micro-perturbations, comme celles observées dans les coupes. Là encore, l'approche stationnelle a établi une échelle d'observation de la flore qui est assez large puisqu'elle s'étend sur plusieurs centaines de mètres carrés et s'avère bien adaptée à l'observation des arbres. Or, les plantes dépendent en partie de conditions variant à une échelle plus fine, comme celles qui découlent des perturbations du sol. Les résultats montrent que les flores de deux carrés d'un mètre peuvent se distinguer très nettement en fonction de l'état de surface du sol. L'explication de la composition floristique des placettes passe donc par une meilleure prise en compte des variations intra-placette. Une hétérogénéité élevée dans une placette produira une flore différente, et sans doute plus riche, que celle d'une placette plus homogène.

Du point de vue de la gestion de la flore forestière, la dimension spatiale et les micro-perturbations nécessitent une attention particulière. La proximité des milieux agricoles et les perturbations du sol facilitent l'arrivée d'espèces non forestières en forêt. Or, certaines d'entre elles, comme par exemple Solidago canadensis, pourraient être parfois envahissantes, poser des problèmes d'allélopathie vis-à-vis des arbres ou d'autres composantes de la flore (Harrod, 2001 ; Jobidon, 1992). Inversement, le développement rapide de la flore peut aussi avoir des effets positifs, par exemple en limitant l'extension d'espèces trop dominantes (Camaret, 1997) ou pour favoriser les processus pédologiques comme cela a été fait en Suède pour réduire le lessivage du sol et l'acidification des cours d'eau (Olsson et Staaf, 1995). À cet égard, les évolutions des techniques d'exploitation forestière durant les dernières décennies, avec aujourd'hui des engins de plusieurs dizaines de tonnes qui circulent aussi bien en été qu'en hiver (Deconchat, 2001), n'ont pas été évaluées jusqu'alors en France pour leurs effets sur la végétation. Des études spécifiques seront sans doute nécessaires dans l'avenir sur ce point.

En guise de conclusion, on peut souligner que ces résultats sont représentatifs de la nécessité d'aborder l'étude des processus écologiques, comme la dynamique de la végétation, à plusieurs échelles spatiales et temporelles (Forman et Godron, 1986). La végétation d'une placette dépend ainsi de facteurs qui agissent à ce niveau (conditions stationnelles notamment), mais aussi à des niveaux plus larges comme l'influence de la proximité des espaces agricoles et plus fins comme l'hétérogénéité de l'état du sol intra-placette. Cette approche à plusieurs échelles, mise en avant 
dans les concepts et méthodes de ce qu'on appelle l'écologie du paysage (même si elle concerne autre chose que les paysages), constitue une méthode utile à la recherche, mais aussi utilisable par les gestionnaires pour identifier l'ensemble des facteurs influant sur la végétation et les hiérarchiser.

Marc DECONCHAT - Gérard BALENT INRA-URSAD-DYNAFOR BP 27

F-31326 CASTANET TOLOSAN CEDEX (marc.deconchat@toulouse.inra.fr) (balent@toulouse.inra.fr)

\section{BIBLIOGRAPHIE}

BALENT (G.). - La Forêt paysanne dans l'espace rural. Biodiversité, paysages, produits. - Études et Recherches sur les Systèmes agraires et le Développement, vol. 29, 1996, pp. 1-268.

BARKHAM (J.P.). - The effects of coppicing and neglect on the performance of the perennial ground flora. In : Ecology and management of coppice woodlands / G.P. Buckley Ed. - London (UK) : Chapman \& Hall, 1992. - pp. 115-146.

BERGQUIST (J.), ORLANDER (G.), NILSSON (U.). - Deer browsing and slash removal affect field vegetation on south Swedish clearcuts. - Forest Ecology and Management, vol. 115, n 2-3, 1999, pp. 171-182.

CAMARET (S.). - Rôle des perturbations dans la dynamique des pessières d'altitude. Impact d'ouvertures artificielles et naturelles sur la régénération et le développement d'un peuplement forestier. - Université de Savoie, 1997.

CHEVALIER (R.), BERGÈS (L.), GILBERT (J.-M.), GOSSELIN (F.). - Station forestière et végétation spontanée d'accompagnement : perspectives pour la diversification des jeunes pineraies de l'Orléanais. - Revue forestière française, vol. LIII, $\mathrm{n}^{\circ}$ 2, 2001, pp. 151-170.

DECONCHAT (M.). - Exploitation forestière et biodiversité : exemple dans les forêts fragmentées des coteaux de Gascogne. - Université Toulouse III, 1999. - 191 p.

DECONCHAT (M.). - Effets des techniques d'exploitation forestière sur l'état de surface du sol. - Annals of Forest Sciences, vol. 58, no 3, 2001, pp. 653-661.

DECONCHAT (M.), BALENT (G.). - Effets des perturbations du sol et de la mise en lumière occasionnées par l'exploitation forestière sur la flore à une échelle fine. - Annals of Forest Sciences, vol. 58, $\mathrm{n}^{\circ}$ 3, 2001a, pp. $315-328$.

DECONCHAT (M.), BALENT (G.). - Vegetation and bird community dynamics in fragmented coppice forests. - Forestry, vol. 74, no 2, 2001b, pp. 105-118.

DECONCHAT (M.), BALENT (G.), VIEBAN (S.), LAUGA-REYREL (F.). - Relations entre biodiversité et exploitation forestière : bases pour une méthode d'analyse. - Revue forestière française, vol. L, n² 2, 1998, pp. 159168.

DUPOUEY (J.-L.), SCIAMA (D.), KOERNER (W.), DAMBRINE (E.), RAMEAU (J.-C.). - La Végétation des forêts anciennes. - Revue forestière française, vol. LIV, $\mathrm{n}^{\circ} 6$ spécial "La végétation forestière : gestion, enjeux et évolution", 2002, pp. 521-532.

FORMAN (R.T.T.), GODRON (M.). - Landscape Ecology. - New York (USA) : John Wiley \& Sons, 1986. $610 \mathrm{p}$.

FROCHOT (H.), ARMAND (G.), GAMA (A.), NOUVEAU (M.), WEHRLEN (L.). - La Gestion de la végétation accompagnatrice : état et perspective. - Revue forestière française, vol. LIV, $\mathrm{n}^{\circ} 6$ spécial "La végétation forestière : gestion, enjeux et évolution", 2002, pp. 505-520.

HALPERN (C.B.), SPIES (T.A.). - Plant species diversity in natural and managed forests of the Pacific Northwest. - Ecological Applications, vol. 5, 1995, pp. 913-934. 
HARROD (R.J.). - The effect of invasive and noxious plants on land management in eastern Oregon and Washington. - Northwest Science, vol. 75, 2001, pp. 85-90.

JOBIDON (R.). - Allelopathy in Quebec forestry -case studies in natural and managed ecosystems. In : Allelopathy : Basic and applied aspects / S.J.H. Rizvi, V. Rizvi Eds. - London (UK) : Chapman \& Hall, 1992. - pp. 342-356.

KOBAYASHI (T.), HORI (Y.), NOMOTO (N.). - Effects of trampling and vegetation removal on species diversity and micro-environment under different shade conditions. - Journal of Vegetation Science, vol. 8, 1997, pp. 873-880.

LE TACON (F.), SELOSSE (M.-A.), GOSSELIN (F.). - Biodiversité, fonctionnement des écosystèmes et gestion forestière. Deuxième partie : Interventions sylvicoles et biodiversité. - Revue forestière française, vol. LIII, $\mathrm{n}^{\circ}$ 1, 2001, pp. 55-80.

MOU (P.), FAHEY (T.J.), HUGHES (J.W.). - Effects of soil disturbance on vegetation recovery and nutrient accumulation following whole-tree harvest of a northern hardwood ecosystem. - Journal of Applied Ecology, vol. 30, 1993, pp. 661-675.

OLSSON (B.A.), STAAF (H.). - Influence of harvesting intensity of logging residues on ground vegetation in coniferous forests. - Journal of Applied Ecology, vol. 32, 1995, pp. 640-654.

RAMEAU (J.-C.), TIMBAL (J.). - Protection de la flore et foresterie. - Revue forestière française, vol. XXXIX, $\mathrm{n}^{\circ} 1,1987$, pp. 26-32.

VAN DER WERF (S.). - The influence of coppicing on vegetation. - Vegetatio, vol. 92, $\mathrm{n}^{\circ}$ 2, 1991, pp. 97110.

\section{EFFETS DE LA SYLUICULTURE ET DE L'EYPLOITRTION FORESTIÈRE SUR LA DIUERSITE UÉGÉthLE [Résumé]}

Les résultats de deux études sur les taillis et leur exploitation montrent que, à l'occasion des interventions sylvicoles et outre l'effet de la station, la flore est soumise d'une part à l'influence spatiale de la plus ou moins grande proximité des espaces agricoles qui modifient la dynamique de la végétation après une coupe et, d'autre part, aux micro-perturbations de la surface du sol occasionnées par l'exploitation forestière. Ces facteurs ont été peu étudiés jusqu'alors, bien qu'ils puissent influer fortement sur la flore ; leur prise en compte semble une nécessité dans la perspective de changements bioclimatiques et d'évolution des pratiques forestières.

\section{EFFECTS OF FORESTRY PAACTISES AND LOGGING ON PLANT DIUERSITY [Abstract]}

The results of two studies on coppice systems and logging thereof show that when silvicultural practices are implemented, in addition to the site effect, the flora is also subject to 1) the spatial influence of the distance from cultivated areas which affects the dynamics of the vegetation following felling and, 2) micro-disturbances of the soil surface caused by logging operations. So far, little attention had been given to these factors although they may significantly impact the flora. They deserve to be considered in view of expected global changes and modified forestry practises. 